\title{
Connected and Total Vertex covering in Graphs
}

\author{
Sathikala $L^{a}$, Basari Kodi $K^{b}$, and Subramanian $K^{c}$
}

a,b,c

Department of Mathematics, Ramco Institute of Technology, Rajapalayam, India

Article History: Received: 11 January 2021; Accepted: 27 February 2021; Published online: 5 April 2021

Abstract: A Subset S of vertices of a Graph G is called a vertex cover if S includes at least one end point of every edge of the Graph. A Vertex cover $S$ of $G$ is a connected vertex cover if the induced subgraph of $S$ is connected. The minimum cardinality of such a set is called the connected vertex covering number and it is denoted by . A Vertex cover S of $\mathrm{G}$ is a total vertex cover if the induced subgraph of $\mathrm{S}$ has no isolates. The minimum cardinality of such a set is called the total vertex covering number and it is denoted by .In this paper a few properties of connected vertex cover and total vertex covers are studied and specific values of and of some well-known graphs are evaluated.

\section{Keywords: Vertex cover, connected vertex cover, Total vertex}

\section{Introduction}

By a graph $G=(V, E)$ we mean a finite, undirected and connected graph with neither self loops nor multiple edges. The order and size of $G$ are denoted by n and m respectively. For graph theoretic terminology, we refer to Chartrand and Lesniak [1].

We start with following definitions and theorems.

1. A subset $S$ of vertices of a graph $G$ is called a vertex cover if $S$ includes at least one end point of every edge of the graph and the minimum cardinality of such a vertex cover is called vertex covering number and it is denoted by $\alpha(G)$.

2. A subset $\mathrm{S}$ of vertices of a graph $\mathrm{G}$ is called a dominating set of a graph if each vertex not in $S$ is adjacent with some vertex in S. The domination number $\gamma(G)$ is the minimum cardinality of a dominating set of G.

3. A dominating set $\mathrm{S}$ of a connected graph is called connected dominating set if the induced subgraph of $\mathrm{S}$ is connected and the minimum cardinality of such a set is called connected domination number and it is denoted by $\gamma_{c}(G)$.

4. A Total dominating set $\mathrm{S}$ of a graph $\mathrm{G}$ is a dominating set in which the induced subgraph of $S$ has no isolates and the minimum cardinality of such a set is called the Total domination number and it is denoted by $\gamma_{t}(G)$.

5. A set $\mathrm{S}$ of vertices in a graph is said to be an independent set if no two vertices in $\mathrm{S}$ are adjacent. A maximal independent set is an independent set to which no other vertex can be added to it without destroying its independence property. The number of vertices in the largest independent set is called the independence number and it is denoted by $\beta(G)$

6. A property $\mathrm{P}$ of a set of vertices is said to be hereditary if whenever a set $\mathrm{S}$ has property $\mathrm{P}$, so does every proper subset $S^{\prime} \subset S$. A property $\mathrm{P}$ is super hereditary if whenever a set $\mathrm{S}$ has property $\mathrm{P}$, so does every proper superset $S^{\prime} \supset S$.

7. Let $\mathrm{P}$ denote an arbitrary property of a set of vertices $\mathrm{S}$ in a graph $G=(V, E)$. If a set $\mathrm{S}$ has property $\mathrm{P}$, then we say that $\mathrm{S}$ is a $\mathrm{P}$-set; otherwise it is a $\bar{P}_{\text {-set. }}$

8. A P-set $\mathrm{S}$ is a maximal P-set if every proper super set $S^{\prime} \supset S$ is a $\bar{P}$-set that is $S^{\prime}$ does not have property P. A P-set $\mathrm{S}$ is a 1-maximal P-set if for every vertex $u \in V-S, S \cup\{u\}$ is a $\bar{P}$-set.

9. A P-set $\mathrm{S}$ is a minimal P-set if every proper subset $S^{\prime} \subset S$ is a $\bar{P}$-set. A P-set $\mathrm{S}$ is a 1 -minimal P-set if for every vertex $v \in S, S-\{v\}$ is a $\bar{P}_{\text {-set. }}$

10. A matching is any independent set of edges. A maximal matching is a matching in $X$ so that $V-V(X)$ is an independent set of vertices. 
11. A perfect matching in a graph $\mathrm{G}$ is a matching $\mathrm{X}$ so that $\mathrm{V}(\mathrm{X})=\mathrm{V}(\mathrm{G})$.Let $\beta_{1}(G)$ denote the size of a maximum in $\mathrm{G}$. The number of edges in a smallest maximal independent set of edges in $\mathrm{G}$ is denoted by $\beta_{1}^{\prime}(G)$

12. A split graph is a graph in which the vertices can be partitioned into a clique and an independent set.

Cockayne et al [2] obtained the following fundamental results for hereditary and super hereditary properties.

Proposition 1.1 [2] Let $G=(V, E)$ be a graph and let $\mathrm{P}$ be a hereditary property. Then a set $\mathrm{S}$ is maximal $\mathrm{P}$ set if and only if $\mathrm{S}$ is a 1-maximal P-set.

Proposition 1.2 [2] Let $G=(V, E)$ be a graph and let $\mathrm{P}$ be a super hereditary property. Then a set $\mathrm{S}$ is minimal $\mathrm{P}$-set if and only if $\mathrm{S}$ is a 1-minimal $\mathrm{P}$-set.

Theorem 1.3 [3] For any graph $\mathrm{G}$ of order $\mathrm{n}$, then $\alpha(G)+\beta(G)=n$.

A vertex cover $\mathrm{S}$ of $\mathrm{G}$ is a connected vertex cover if the induced subgraph of $\mathrm{S}$ is connected. The minimum cardinality of such a set is called the connected vertex covering number and it is denoted by $\alpha_{c}(G)$.

A vertex cover $\mathrm{S}$ of $\mathrm{G}$ is a total vertex cover if the induced subgraph of $\mathrm{S}$ has no isolates. The minimum cardinality of such a set is called the total vertex covering number and it is denoted by $\alpha_{t}(G)$.

In this paper a few properties of connected vertex cover and total vertex covers are studied and specific values of $\alpha_{c}(G)$ and $\alpha_{t}(G)$ of some well-known graphs are evaluated.

Many variants of connected domination and total domination number have been already studied.

\section{Connected and Total Vertex Covering In Graphs}

The property of being a connected vertex cover is a super hereditary property. Hence a connected vertex cover is minimal if and only if it is 1-minimal.

Theorem 2.1

A connected vertex covering $\mathrm{S}$ is a minimal connected vertex covering if and only if for each $v \in S$, one of the following holds.

(a) 'v' is a cut vertex in $\langle S\rangle$

(b) There exists a vertex $u \in V-S$ such that $u v \in E(G)$.

Proof:

Let $S$ be a minimal connected vertex covering of a graph G. Then for every vertex $v \in S, S-\{v\}$ is not a connected vertex covering. This means that either $\langle S-\{v\}\rangle$ is not connected or an edge in $V-S$ is not covered by $S-\{v\}$. This implies that either $\mathrm{v}$ is a cut vertex in $\langle S\rangle$ or there exists a vertex $u \in V-S$ such that $u v \in E(G)$

Conversely suppose that $\mathrm{S}$ is a connected vertex covering of a graph $\mathrm{G}$ and for each $v \in S$, one of the stated conditions hold. We show that $S$ is a minimal connected vertex covering of a graph. Suppose $S$ is not a minimal connected vertex covering, then there exists a vertex $v \in S$ such that $S-\{v\}$ is a connected vertex covering. Hence $\langle S-\{v\}\rangle$ is connected and $\mathrm{v}$ is not a cut vertex of $\langle S\rangle$. Also if $S-\{v\}$ is a connected vertex covering, then there is no edge in $V-\{S-\{v\}\}$ which is not covered by $S-\{v\}$. This means that there is no vertex $u \in V-S$ such that $u v \in E(G)$. Hence $\mathrm{S}$ is a minimal connected vertex covering of a graph $\mathrm{G}$.

We observe that the property of being a total vertex covering is a super hereditary property. Hence the total vertex covering $\mathrm{S}$ is minimal if and only if it is 1 -minimal.

Theorem 2.2

A Total vertex covering $\mathrm{S}$ is a minimal total vertex covering if and only if for each $u \in S$, one of the following conditions hold:

(a) there exists a vertex $w \in S$ such that $N(w) \cap S=\{u\}$.

(b) there exists a vertex $v \in V-S$ such that $u v \in E(G)$.

Proof

Let $\mathrm{S}$ be a minimal total vertex covering of a graph G. Then for every $u \in S, S-\{u\}$ is not a total vertex covering. This means that $\langle S-\{u\}\rangle$ has an isolate or an edge in $(V-S) \cup\{u\}$ is not covered by $S-\{u\}$. This 
means that there exists a vertex $w \in S$ such that $N(w) \cap S=\{u\}$ or there exists a vertex $v \in V-S$ such that $u v \in E(G)$

Conversely suppose that $\mathrm{S}$ is a total vertex covering and for each $u \in S$, one of the stated conditions hold. We show that $\mathrm{S}$ is a minimal total vertex covering of a graph $\mathrm{G}$. Suppose $\mathrm{S}$ is not a minimal total vertex covering, then there exists a vertex $u \in S$ such that $S-\{u\}$ is a total vertex covering and hence $S-\{u\}$ has no isolates. Then there is no vertex $w \in S$ such that $N(w) \cap S=\{u\}$. Also if $S-\{u\}$ is a total vertex covering, then there is no edge in $(V-S) \cup\{u\}$ which is not covered by $S-\{u\}$ and hence there is no vertex $v \in V-S$ such that $u v \in E(G)$. Hence $S$ is a minimal total vertex covering of a graph $\mathrm{G}$.

Observations 2.3

(i) $\alpha_{c}(G)=1$ if and only if $\mathrm{G}$ is star.

(ii) Since every connected vertex cover is a connected dominating set, we have $\gamma_{c}(G) \leq \alpha_{c}(G)$.

(iii) Since every total vertex covering is a total dominating set, $\gamma_{t}(G) \leq \alpha_{t}(G)$.

(iv) Since every connected vertex cover is a total vertex cover of $\mathrm{G}, \alpha_{t}(G) \leq \alpha_{c}(G)$.

(v) There is no relation between $\alpha_{t}(G)$ and $\gamma_{c}(G)$.

For example,

If $\mathrm{G}$ is a star $K_{1, n}$, then $\alpha_{t}(G)=2$ and $\gamma_{c}(G)=1$.

$$
\text { Here } \alpha_{t}(G)>\gamma_{c}(G) \text {. }
$$

If $\mathrm{G}$ is a path of length 10 , then $\alpha_{t}(G)=6$ and $\gamma_{c}(G)=8$.

Here $\alpha_{t}(G)<\gamma_{c}(G)$.

Hence there is no relation between $\alpha_{t}(G)$ and ${ }^{\gamma_{c}}(G)$.

Theorem 2.4

For any graph $\mathrm{G}, \alpha_{c}(G)+\beta_{0}(G) \geq n$.

\section{Proof}

Let $\mathrm{S}$ be a connected vertex covering of $\mathrm{G}$. Then $\mathrm{S}$ is a vertex cover of $\mathrm{G}$ implies that $V-S$ is independent.

Thus $|V-S| \leq \beta_{0}(G)$

$\Rightarrow n-\alpha_{c}(G) \leq \beta_{0}(G)$

$\alpha_{c}(G)+\beta_{0}(G) \geq n$

We illustrate this with an example. Consider the complete bipartite graph $K_{4,5}$.

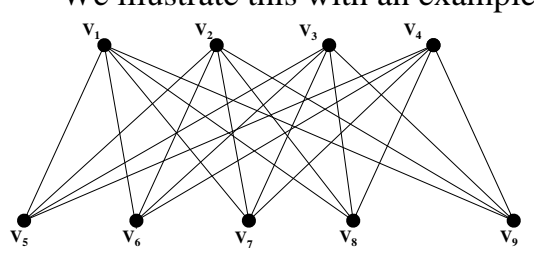

Here $\alpha_{c}(G)=5, \beta_{0}(G)=5$.

Thus $\alpha_{c}(G)+\beta_{0}(G)=5+5=10 \geq 9$.

Hence $\alpha_{c}(G)+\beta_{0}(G) \geq n$.

Observations 2.5

We have $\beta_{0}(G)=n-\delta(G)$ where $\delta$ is the minimum degree of graph G.

Thus $\alpha_{c}(G)+\beta_{0}(G) \geq n$

$\Rightarrow \alpha_{c}(G) \geq n-\beta_{0}(G)=n-(n-\delta)=\delta(G)$

Hence for any connected graph we have 


$$
\delta(G) \leq \alpha_{c}(G) \leq n-1
$$

Both the bounds are sharp. $K_{1, n}$.

We observe that $\alpha_{c}(G)=n-1$ if $\mathrm{G}$ is a complete graph or cycle on 'n' vertices and $\alpha_{c}(G)=\delta$ if G is a star

\section{Theorem 2.6}

Let $a$ and $b$ be two positive integers such that $2 \leq a \leq b$. Then there exists a graph $\mathrm{G}$ with $\gamma_{c}(G)=a, \alpha_{c}(G)=b$

\section{Proof}

Case (i): $a=b$

Let us take a path on ' $a$ ' vertices denoted by ${ }^{a}$. Consider corona of path $P_{a}$. That is $P_{a} \circ K_{1}$. For this graph $\gamma_{c}(G)=a, \alpha_{c}(G)=b=a$

Case (ii): $a<b$

Consider the path on ' $a$ ' vertices say $P_{a}=\left(v_{1}, v_{2}, \ldots v_{a}\right)$. Attach $2(b-a)$ pendent vertices to ${ }^{v_{1}}$ and let it be $u_{1}, u_{2}, \ldots u_{2(b-a)}$ and attach two pendents to all other vertices and then join the vertices $u_{1}, u_{2}, u_{3}, u_{4}$ and $u_{5}, u_{6}, \ldots u_{2(b-a)-1}, u_{2(b-a)}$.

Clearly $\gamma_{c}(G)=a, \alpha_{c}(G)=a+(b-a)=b$

Let us illustrate this construction with examples.

Example (1): Take $a=b=4$. Then $P_{4} \circ K_{1}$ is

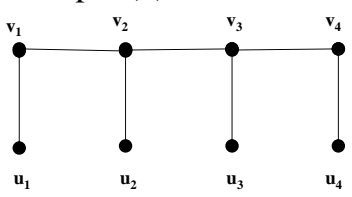

$$
\text { Here } \gamma_{c}(G)=4, \alpha_{c}(G)=4
$$

Example (2): Take $a=5, b=8$.

Then $P_{5}$ is be

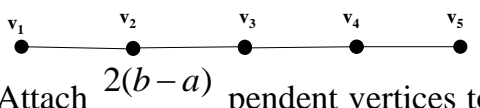

Attach $2(b-a)$ pendent vertices to $\mathrm{v}_{1}$ and two pendent vertices to all other vertices. Then the graph $\mathrm{G}$ will

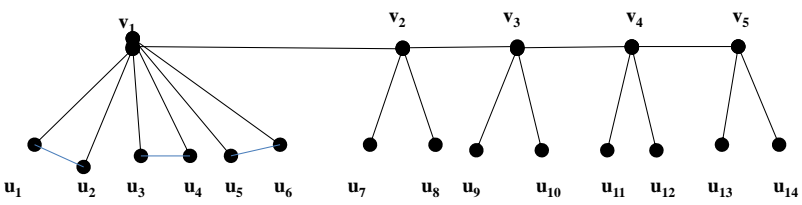

Clearly $\gamma_{c}(G)=5, \alpha_{c}(G)=5+3=8$

$\alpha_{c}(G)$ AND $\alpha_{t}(G)$ FOR SOME GRAPHS

(1) For complete graph $K_{n}$,

$$
\alpha_{c}(G)=n-1, \alpha_{t}(G)=n-1 \text {. }
$$

(2) For star $K_{1, n}, \alpha_{c}(G)=1, \alpha_{t}(G)=2$.

(3) For Bistar $K_{n_{1}, n_{2}}, \alpha_{c}(G)=2, \alpha_{t}(G)=2$

Next, we characterise graphs for which $\alpha_{c}(G)=2$ and $\alpha_{t}(G)=2$. Theorem 3.1 
For any graph $\mathrm{G}$ of order $\mathrm{n}, \alpha_{c}(G)=2$ if and only if $\mathrm{G}$ is a split graph with the split partition $\mathrm{S}$ and V-S such that $|S|=2,|V-S|=n-2$ with $\langle S\rangle=K_{2}$ and $\langle V-S\rangle$ is independent.

\section{Proof}

$$
\text { Suppose } \alpha_{c}(G)=2 \text {. }
$$

Let $\mathrm{S}$ be a connected vertex covering of $\mathrm{G}$ with 2 vertices. Since $\mathrm{S}$ is connected, $\mathrm{S}$ is isomorphic to $\mathrm{K}_{2}$. Since $\mathrm{S}$ is a vertex cover, $\mathrm{V}-\mathrm{S}$ is independent. Hence $\mathrm{G}$ is isomorphic to a split graph with split partition $\mathrm{S}$ and $\mathrm{V}-\mathrm{S}$ such that $\langle S\rangle=K_{2}$ and $\langle V-S\rangle$ is independent and converse is obvious.

Similarly, we have the following Theorem.

Theorem 3.2

For any graph $\mathrm{G}$ of order $\mathrm{n}, \alpha_{t}(G)=2$ if and only if $\mathrm{G}$ is a split graph with the split partition $\mathrm{S}$ and V-S such that $|S|=2,|V-S|=n-2$ with $\langle S\rangle=K_{2}$ and $\langle V-S\rangle$ is independent.

Open Problems

1. Characterise graphs for which $\alpha_{c}(G)=\alpha_{t}(G)$.

2. Obtain upper bounds for $\alpha_{c}(G)$ and $\alpha_{t}(G)$ for special types of graphs like Trees, Petersen graph, etc.

3. Find bounds for $\gamma_{c}(G)$ and $\alpha_{t}(G)$ ?

\section{References}

1. Min Li, et al, vol 8, 2020, "Computer-Aided Diagnosis and Staging of Pancreatic Cancer Based on CT Images”, IEEE Access, pp: 141705 - 141718.

2. HaswanthVundavilli, et al, vol 17, 2020, "In Silico Design and Experimental Validation of Combination Therapy for Pancreatic Cancer”, IEEE/ACM Transactions on Computational Biology and Bioinformatics, pp:1010 - 1018 .

3. XiaojunYu, et al, vol 25, 2019, "Evaluating Micro-Optical Coherence Tomography as a Feasible Imaging Tool for Pancreatic Disease Diagnosis”, IEEE Journal of Selected Topics in Quantum Electronics, Seq no:6800108.

4. Zhitong Chen, et al, vol 2, 2018, "Selective Treatment of Pancreatic Cancer Cells by Plasma-Activated Saline Solutions", IEEE Transactions on Radiation and Plasma Medical Sciences, pp:116 - 120.

5. Sarfaraz Hussein, et al, vol 38, 2019, "Lung and Pancreatic Tumor Characterization in the Deep Learning Era: Novel Supervised and Unsupervised Learning Approaches”, IEEE Transactions on Medical Imaging, pp: $1777-1787$.

6. Ken C. L. Wong, et al, vol 36, 2017, "Pancreatic Tumor Growth Prediction With Elastic-Growth Decomposition, Image-Derived Motion, and FDM-FEM Coupling”, IEEE Transactions on Medical Imaging, pp: $111-123$.

7. Brooks D. Lindsey, et al, vol 64, 2017, "Dual-Frequency Piezoelectric Endoscopic Transducer for Imaging Vascular Invasion in Pancreatic Cancer”, IEEE Transactions on Ultrasonics, Ferroelectrics, and Frequency Control, pp: 1078 - 1086.

8. Phuong Vincent, et al, vol 67, 2020, "High-Resolution Ex Vivo Elastography to Characterize Tumor Stromal Heterogeneity In Situ in Pancreatic Adenocarcinoma”, IEEE Transactions on Biomedical Engineering, pp: $2490-2496$.

9. Shangqing Liu, et al, vol 8, 2019, “Automatic Pancreas Segmentation via Coarse Location and Ensemble Learning”, IEEE Access, pp: 2906 - 2914.

10. LingxiXie, et al, vol 39, 2019, "Recurrent Saliency Transformation Network for Tiny Target Segmentation in Abdominal CT Scans", IEEE Transactions on Medical Imaging, pp: 514 - 525.

11. Ling Zhang, et al, vol 37, 2018, “Convolutional Invasion and Expansion Networks for Tumor Growth Prediction", IEEE Transactions on Medical Imaging, pp: $638-648$.

12. Senthil Kumar Chinnaiyan et al, vol 13, 2019, "5 Fluorouracil-loaded biosynthesised gold nanoparticles for the in vitro treatment of human pancreatic cancer cell”, IET Nano-biotechnology, pp: $824-828$.

13. Yiqun Du et al, vol 13, 2019, "PEGylated zinc oxide nanoparticles induce apoptosis in pancreatic cancer cells through reactive oxygen species", IET Nanobiotechnology, pp: 536 - 540.

14. A.Sheryl Oliver,M.Anuratha,M.Jean Justus,Kiranmai Bellam, T.Jayasankar, "An Efficient Coding Network Based Feature Extraction with Support Vector Machine Based Classification Model for CT Lung Images,” J. Med. Imaging Health Inf. ,vol.10,no.11.pp.2628-2633(2020). 
15. YashwantKurmi et al, vol 14, 2020, "Classification of magnetic resonance images for brain tumour detection", IET Image Processing, pp: 2808 - 2818.

16. Neelum Noreen et al, vol 8, 2020, “A Deep Learning Model Based on Concatenation Approach for the Diagnosis of Brain Tumor”, IEEE Access, pp: 55135 - 55144.

17. J.Jayanthi, T.Jayasankar, N.Krishnaraj, N.B.Prakash, A.Sagai Francis Britto, K.Vinoth Kumar, "An Intelligent Particle Swarm Optimization with Convolutional Neural Network for Diabetic Retinopathy Classification Model, " Journal of Medical Imaging and Health Informatics (2020), Volume 11, Number 3, March 2021, pp. 803-809, https://doi.org/10.1166/jmihi.2021.3362

18. M.Anuradha, T.Jayasankar, PrakashN.B ${ }^{3}$, Mohamed Yacin Sikkandar, G.R.Hemalakshmi, C.Bharatiraja,A. Sagai Francis Britto, "IoT enabled Cancer Prediction System to Enhance the Authentication and Security using Cloud Computing," Microprocessor and Microsystems (Elsevier 2021), vol 80, February, (2021) .https://doi.org/10.1016/j.micpro.2020.103301

19. Ping Liu;QiDou;QiongWang;Pheng-AnnHeng, Year: 2020, “An Encoder-Decoder Neural Network With 3D Squeeze-and-Excitation and Deep Supervision for Brain Tumor Segmentation”, IEEE Access, Vol: 8, pp:34029 - 34037 .

20. Mhd Saeed Sharif;MaysamAbbod;AliAl-Bayatti;AbbesAmira;Ahmed S.Alfakeeh; BalSanghera, Year: 2020, "An Accurate Ensemble Classifier for Medical Volume Analysis: Phantom and Clinical PET Study", IEEE Access, Vol: 8, pp: 37482 - 37494.

21. Kavitha R.J., Avudaiyappan T., Jayasankar T., Selvi J.A.V. (2021) Industrial Internet of Things (IIoT) with Cloud Teleophthalmology-Based Age-Related Macular Degeneration (AMD) Disease Prediction Model. In: Gupta D., Hugo C. de Albuquerque V., Khanna A., Mehta P.L. (eds) Smart Sensors for Industrial Internet of Things. Internet of Things (Technology, Communications and Computing). Springer, Cham.pp.161-172 https://doi.org/10.1007/978-3-030-52624-5_11. 